\title{
AUDITORIA DE LA GESTIÓN EMPRESARIAL
}

\section{AUDITING BUSINESS MANAGEMENT}

\section{JUAN GUILLERMo Miñano LeCARos*}

Docente Asociado de la Facultad de Ciencias Contables - UNMSM

[Recepción: Setiembre de 2011/ Conformidad: Noviembre 2011]

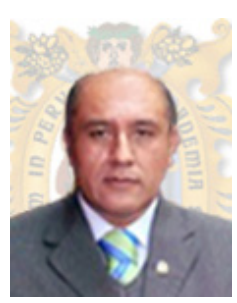

\section{RESUMEN}

El presente artículo destaca la importancia del control de gestión entendida esta como el análisis de eficiencia y eficacia de las entidades de administración y de los recursos públicos, determinada mediante la evaluación de los procesos administrativos, la utilización de indicadores de rentabilidad pública y desempeño.

La Auditoría de Gestión permite abarcar el crecimiento constante de problemas cada vez más complejos ya que motivado por las exigencias sociales y de la tecnología pasa a ser un elemento vital para la gerencia, permitiéndole conocer a los ejecutivos qué tan bien resuelven los problemas económicos, sociales que a este nivel se presentan generando en la empresa un saludable dinamismo.

La Auditoría de gestión o el Control de gestión como cualquier sistema, tiene instrumentos que sirven de ayuda para las entidades, por lo que aquí determinaremos la importancia de cada uno de ellos: Cuadros de mandos, indicadores, análisis comparativo, control integral, etc.

Palabras clave: Auditoría de Gestión, Gestión empresarial, Control Integral, Control de gestión.

\begin{abstract}
This article highlights the importance of management control understood as the analysis of efficiency and effectiveness of government institutions and public resources, it's determined through the evaluation of administrative processes, the use of public performance indicators and performance.

The Auditing allows constant growth include increasingly complex problems as motivated by social demands and technology becomes a vital element for management, enabling managers to know how well solve economic and social problems that at this level are presented in the company generating a healthy dynamic.

Audit of management or any management control system, has tools that are helpful for entities, so here it will be determined the important of each one of them: Scorecards, indicators, benchmarking, integrated control, etc...
\end{abstract}

Keywords: Auditing, Business Management, Integral Control, Management Control.

* Doctor en Ciencias Contables y Empresariales - UNMSM. Docente Asociado de la Facultad de Ciencias Contables UNMSM. Docente Investigador de la Facultad de Ciencias Contables -UNMSM. Actual Director de la Unidad d Postgrado - UNMSM. 


\section{INTRODUCCIÓN}

La Auditoría de Gestión surge recientemente y como resultado del desarrollo social, del avance tecnológico y la práctica productiva en un mundo cada vez más globalizado sin perder de vista que en esta auditoría se da una contradicción no antagónica que es esencial: lo general que se establece por los organismos competentes, para poder desenvolverse en este campo y lo particular que deben tener estas auditorías a partir de las características estructurales y funcionales de cada objeto de investigación. Se evalúa la gestión que se realiza en la planificación, control y uso de los recursos y se comprueba la observancia de las disposiciones pertinentes para verificar su racional utilización y contribuir a mejorar las actividades y materias examinadas. Lograr, a través de ella que los ejecutivos evalúen la efectividad de los procedimientos seguidos y si estos están dirigidos a cumplir los objetivos fijados.

Muchas son las causas que hacen insuficiente el número de las auditorías de gestión que se ejecutan. Los directivos de las empresas no reconocen en esta auditoría una aliada en el logro de los objetivos económicos, sociales y ambientales que ellos deben cumplir. Los auditores se ven y son vistos como los "buscadores de fraudes y errores" de viejos paradigmas. Es evidente que existe están desfasados en el tiempo los cambios económicos - sociales que se promueven y el desarrollo de la Auditoría de Gestión, estando entre las principales causas: Poca cultura de los ejecutivos sobre esta auditoría y sus ventajas.

\section{USO DE INDICADORES EN AUDITORIA DE GESTIÓN}

El uso de INDICADORES en la Auditoria, permite medir:
- La eficiencia y economía en el manejo de los recursos

- Las cualidades y características de los bienes producidos o servicios prestados (eficacia).

- El grado de satisfacción de las necesidades de los usuarios o clientes a quienes van dirigidos (calidad).

\section{PARÁMETROS E INDICADORES DE LA AUDITORÍA DE GESTIÓN}

Para conocer y aplicar los indicadores de gestión, es importante que se encuentre implementado el control interno en la entidad y que se tenga muy en claro lo que es y la importancia del control de gestión como concepto clave y herramienta de la evaluación institucional.

Uno de los resultados del control interno, que permite ir midiendo y evaluando es el control de gestión, que a su vez pretende eficiencia y eficacia en las instituciones a través del cumplimiento de las metas y objetivos, tomando como parámetros los llamados indicadores de gestión, los cuales se materializan de acuerdo a las necesidades de la institución, es decir, con base en lo que se quiere medir, analizar, observar o corregir.

Las actividades que se realizan en una organización requieren cuantificarse, es decir, medirse; esa medida refleja en qué grado las actividades que se ejecutan dentro de un proceso, o los resultados del proceso se han alcanzado. Para medir una actividad lo importante es saber:

Para medir una actividad lo importante es saber: Indicadores cuantitativas y cualitativos.

Los indicadores desde el punto de vista de instrumentos de gestión, son de dos tipos: 
a) Indicadores cuantitativos, que son los valores y cifras que se establecen periódicamente de los resultados de las operaciones, son un instrumento básico.

b) Indicadores cualitativos, que permiten tener en cuenta la heterogeneidad, las amenazas y las oportunidades del entorno organizacional; permite además evaluar, con un enfoque de planeación estratégica la capacidad de gestión de la dirección y de más niveles de la organización.

\section{LAS TÉCNICAS MÁS UTILIZADAS EN LA AUDITORÍA DE GESTIÓN}

En la auditoria de gestión es fundamental el criterio profesional del auditor para la determinación de la utilización y combinación de las técnicas y prácticas más adecuadas, que le permitan la obtención de la evidencia suficiente, competente y pertinente, que le den una base objetiva y profesional, que fundamenten y sustenten sus comentarios, conclusiones y recomendaciones. Se considera importante a continuación definir las técnicas y las prácticas que pueden ser más utilizadas en este tipo.

Comparación.- Es la determinación de la similitud o diferencias existentes en dos o más hechos u operaciones; mediante esta técnica se compara las operaciones realizadas por la entidad auditada, o los resultados de la auditoria con criterios normativos, técnicos y prácticas establecidas, mediante lo cual se puede evaluar y emitir un informe al respecto. Fases: Ejecución y Comunicación de Resultados.

Observación.- Es la verificación visual que realiza el auditor durante la ejecución de una actividad o proceso para examinar aspectos físicos, incluyendo desde la observación del flujo de trabajo, documentos, materiales, etc. Fases: 1 Conocimiento preliminar, 2 Planificación y 3 Ejecución.

Rastreo.- Es el seguimiento y control de una operación, dentro de un proceso o de un proceso a otro, a fin de conocer y evaluar su ejecución. Fases: 1 Conocimiento Preliminar y 3 Ejecución.

Indagación.- Es la obtención de información verbal mediante averiguaciones o conversaciones directas con funcionarios de la entidad auditada o terceros sobre las operaciones que se encuentran relacionadas, especialmente de hechos o aspectos no documentados. Fases: 1 Conocimiento Preliminar y 3 Ejecución.

Encuesta.- Encuestas realizadas directamente o por correo, con el propósito de recibir de los funcionarios de la entidad auditada o de terceros, una información de un universo, mediante el uso de cuestionarios cuyos resultados deben ser posteriormente tabulados. Fase: 3 Ejecución.

Análisis.- Consiste en la separación y evaluación crítica, objetiva y minuciosa de los elementos o partes que conforman una operación, actividad, transacción o proceso, con el propósito de establecer su propiedad y conformidad con criterios normativos y técnicos; como por ejemplo, el análisis de la documentación interna y externa de la entidad hasta llegar a una deducción lógica; involucra la separación de las diversas partes y determina el efecto inmediato o potencial. Fases: 1 Conocimiento Preliminar, 2 Planificación, 3 Ejecución y 5 Seguimiento.

Conciliación.- Consiste en hacer que concuerden dos conjuntos de datos relacionados, separados e independientes; por ejemplo, analizar la información producida por las diferentes unidades operativas o administrativas, con el objeto de hacerles concordantes entre sí y a la vez determinar la 
validez y veracidad de los registros, informes y resultados bajo examen. Fase: 3 Ejecución.

Confirmación.- Comunicación independiente y por escrito, de parte de los funcionarios que participan o ejecutan las operaciones, y/o de fuente ajena a la entidad auditada, para comprobar la autenticidad de los registros y documentos sujetos al examen, así como para determinar la exactitud o validez de una cifra, hecho u operación. Fase: 3 Ejecución.

Comprobación.- Consiste en verificar la existencia, legalidad, autenticidad y legitimidad de las operaciones realizadas por una entidad, a través del examen de la documentación justificatoria o de respaldo. Fases: 1 Conocimiento Preliminar y 3 Ejecución.

Calculo.- Consiste en la verificación de la exactitud y corrección aritmética de una operación o resultado, presentados en informes, contratos, comprobantes y otros. Fase: 3 Ejecución.

Revisión Selectiva.- Consiste en una breve o rápida revisión o examen de una parte del universo de datos u operaciones, con el propósito de separar y analizar los aspectos que no son normales y que requieren de una atención especial y evaluación durante la ejecución de la auditoria. Fases: 1 Conocimiento Preliminar y 3 Ejecución.

Inspección.- técnica involucra el examen físico y ocular de activos, obras, documentos, valores y otros, con el objeto de establecer su existencia y autenticidad; requiere en el momento de la aplicación la combinación de otras técnicas, tales como: indagación, observación, comparación, rastreo, análisis, tabulación y comprobación. Fase. 3 Ejecución.

\section{PROCEDIMIENTOS}

La auditoría de gestión consiste en las revisiones y evaluación de dos elementos mayores de la administración:
Política de la corporación. Determinación de la existencia, lo adecuado y comprensivo de la política así como el significado de sus instructivos como elementos de control en áreas funcionales. Valorización de los efectos de la ausencia de políticas; o recomendaciones para la adopción o modificación de los instructivos formalizados.

Controles administrativos. Determinación de la existencia, y lo adecuado de controles administrativos u operacionales como tales, y como respaldo a los objetivos de productividad de la gerencia; el grado de cumplimiento en las áreas de funcionabilidad mayor; y la coordinación de controles de operación con los instructivos de la política de la corporación.

Evaluación de los efectos de la acción de controles administrativos u operacionales en áreas significativas, y recomendaciones para la adopción o modificación de tales controles.

$\mathrm{Al}$ iniciar una auditoría de gestión, el auditor prepara datos importantes que le sirven como antecedentes respecto al cliente, entre los cuales se tiene las utilidades históricas, recuperación sobre inversión, clases de productos usuales de distribución, características mercantiles inusitadas en el ramo del mercado, el volumen aproximado de ventas anuales, el activo y la influencia de pedidos a la orden o contratos para la diferencia sobre la planeación y operaciones generales. Estos datos pueden suplir con inspecciones a las plantas y otros servicios, a fin de evaluar las condiciones físicas, y localizar indicios de posibles áreas de problemas.

El auditor también puede aplicar técnicas de análisis financiero para estadísticas de operación, que también puede sugerir áreas de problemas, o condiciones que influencian una recuperación desfavorable sobre la inversión, o estadística de operación adversa. 


\section{CONCLUSIONES}

El adecuado planeamiento de la auditoría de gestión, sin ninguna duda, facilita la determinación de la efectividad, eficiencia y economía de la organización.

La preparación de programas de auditoría, la aplicación de técnicas, pruebas y obtención de evidencias de auditoría permiten la elaboración de hallazgos de auditoría, observaciones, conclusiones y recomendaciones de la organización.

Las medidas correctivas para optimizar la gestión y eficiencia de la organización, sólo se puede llevar a cabo si se analiza e interpreta correctamente el contenido de los informes de la auditoría de gestión.

La supervisión o monitoreo llevadas a cabo en forma permanente $y / o$ puntual asegura el seguimiento de las medidas correctivas adoptadas la organización, hasta obtener la eficiencia y eficacia de la misma.

Mediante la aplicación de la Auditoría de Gestión, es posible realizar ajustes, si fuera el caso, a los planes y programas de la organización para su gerenciamiento óptimo.

\section{REFERENCIAS BIBLIOGRÁFICAS}

1. Aquino, Jorge A.; Vola, Roberto E.; Arecco, Marcelo J.; Aquino, Gustavo J. Recursos Humanos. Ediciones Macchi. Argentina, 1997. 2da Edición.

2. Administración. Segunda Edición. Quinta Parte P- 224.

3. Auditoría de Gestión: Segunda Edición, Ciudad Habana: Editorial pueblo y Educación.

4. Barranco, Francisco J. Planificación Estratégica de Recursos Humanos. Del Marketing Interno a la Planificación. Editorial Pirámide. Madrid. 1993 p 342.

5. BEER M et al. Gestión de Recursos Humanos. Perspectiva de un Gerente General. Ministerio de Trabajo y Seguridad Social, Barcelona, 1989.

6. Bustillo, Carlos. La Gestión de Recursos Humano y la Motivación de las personas. Capital Humano (España). 73: 1728, 1994

7. Chiavenato, Idalberto. Administración de Recursos Humanos Editorial McGraw -Hill. México.1993 p-568 